\title{
Assessment of Microscale Test Methods of Peeling and Splitting along Surface of Thin-Film/Substrates
}

Y. Wei

LNM, Institute of Mechanics, Chinese Academy of Sciences, Beijing 100080, P.R. China

\begin{abstract}
Peel test methods are assessed through being applied to a peeling analysis of the ductile film/ceramic substrate system. Through computing the fracture work of the system using the either beam bend model (BB model) or the general plane analysis model (GPA model), surprisingly, a big difference between both model results is found. Although the BB model can capture the plastic dissipation phenomenon for the ductile film case as the GPA model can, it is much sensitive to the choice of the peeling criterion parameters, and it overestimates the plastic bending effect unable to capture crack tip constraint plasticity. In view of the difficulty of measuring interfacial toughness using peel test method when film is the ductile material, a new test method, split test, is recommended and analyzed using the GPA model. The prediction is applied to a wedge-loaded experiment for Al-alloy double-cantilever beam in literature.
\end{abstract}

\section{INTRODUCTION}

The peel test method has been widely used for the mechanical characterization of adhesion phenomena in various applications involving adhesive joining and thin film technology in industries as diverse as the micro-packaging and micro-electronic industries and the automotive industry. It has received considerable attention in the past several decades, and continues to be the subject of intense scrutiny in a wide range of research areas [1,2]. Most mechanical applications are used to measure the interfacial toughness for such a film/substrate system. However, when adherent, film or substrate, is a ductile material, the measurement of the interfacial fracture toughness using the peel test method becomes much difficult even impossible due to plastic dissipation. Therefore, it is important to separate the plastic dissipation from peel test work. For this purpose, several analytical models have been presented. Two representative models are the beam bend model [3] (BB model) and the more recently proposed general plane analysis model [4] (GPA model). Before the GPA model was presented, the BB model had undergone the much attention [5-8]. By the BB model, the thin film is assumed to undergo the elastic-plastic bending deformation, and plastic dissipation comes only from the plastic bending of film, while the plastic dissipation from crack tip constraint (crack tip "singularity" effect) can not be caught. On the GPA model [4], the plastic dissipation includes two contribution sources of both the plastic bending of film and the crack tip constraint. In the present research, the peel test analytical models mentioned above will be assessed through adopting them to a peeling problem of a ductile film/ceramic substrate system. The steady-state fracture work is computed using the either BB model or the GPA model. Through checking and comparing the results of both models, three fundamental objectives hope to be attained. (1) Work contribution from the plastic dissipation is studied; (2) The limitations of conventionally used models in the peel test methods are investigated; (3) Additionally, a new test method, split test, is introduced and further discussed through comparing with the peel test. The prediction result using the split test is applied to a wedge-loaded experiment for Al-alloy double-cantilever beam [8]. 


\section{PEEL TEST ANALYSIS MODELS}



(a)



(b)

Figure 1. Peel test sketch figure (a) and the simplified the embedded process zone model (b).

The peel test geometry is sketched in figure 1(a). Ductile film is delaminated along the interface under a peel force. Under steady-state peeling condition, the ductile film undergoes a deformation process from elastic, elastic-plastic, elastic unloading, reverse plastic bend, finally to unloading. From energy balance, the total fracture work (per unit crack tip advance, or peel force per unit width) can be dictated as

$$
P=\Gamma_{0}+\Gamma^{P}
$$

where $\Gamma_{0}$ is the interface adhesion energy, $\Gamma^{P}$ is the plastic dissipation. When thin film is elastic material, $\Gamma^{P}=0$, one can obtain $\Gamma_{0}$ value easily by measuring the peel force. However, when thin film is elastic-plastic material, it is difficult to obtain the interface adhesion work using the peel test method. A lot of researches have been presented for the case in past decade. Several models have been proposed to try to separate the plastic dissipation from the total work $[3,4]$. The detail assessments for the models will be given in the following subsections.

\section{Beam bend model for peel test problems}

Thin film deformation during the peeling process is treated as the beam bending under plane strain. The process can be sketched by a moment-curvature relation (Fig.1(a)). Because ceramic substrate has the much higher elastic modulus than that of film, one assumes that the substrate is rigid. The tensile stress-strain relation used to characterize the film material is

$$
\sigma=E \varepsilon, \text { for } \sigma<\sigma_{Y} ; \sigma=\sigma_{Y}\left(\varepsilon / \varepsilon_{Y}\right)^{N} \text {, for } \sigma \geq \sigma_{Y}
$$

The moment-curvature relations can be derived for the cases of elastic bending, elastic-plastic bending and unloading respectively,

$$
\begin{aligned}
& M / M_{0}=\frac{2}{3} \kappa / \kappa_{e}, \text { for elastic bending } \\
& M / M_{0}=\left\{\frac{2}{3}-\frac{2}{N+2} \gamma\right\}\left(\kappa / \kappa_{e}\right)^{-2}+\frac{2}{N+2} \gamma\left(\kappa / \kappa_{e}\right)^{N}, \text { for elastic-plastic bending } \\
& M / M_{0}=\frac{2}{3}\left(\kappa-\kappa_{0}\right) / \kappa_{e}, \quad \text { for elastic unloading bending }
\end{aligned}
$$

where $\kappa_{e}$ is the elastic limit curvature, $M_{e}$ and $M_{0}$ are the elastic and plastic limit moments respectively 
for a ideally-plastic material, and $\gamma$ is a composite material parameter. They are defined as follows

$$
\gamma=2{\sqrt{\frac{1}{3}\left(1-v+v^{2}\right)}}^{1-N}(1-v)^{N}, \quad \kappa_{e}=\frac{2\left(1-v^{2}\right) \sigma_{Y}}{E t \sqrt{1-v+v^{2}}}, \quad M_{0}=\frac{3}{2} M_{e}=\frac{\sigma_{Y} t^{2}}{4 \sqrt{1-v+v^{2}}}
$$

where $t$ is the thin film thickness, $E$ is Young's modulus, $v$ is Poisson's ratio. If define a reverse plastic bending parameter $w_{0}$ which is defined in figure 1(a), one can easily obtain a curvature-rotation relation at crack tip (approximately at point B of figure 1(a)),

$$
\kappa_{B}=\sqrt{\left[1-\cos \left(\Phi-\theta_{\text {tip }}\right)\right](2 P / B)+\left(1-w_{0}\right) \kappa_{0}^{2}}
$$

where $\kappa_{0}$ is the residual curvature just after loading, $B=E t^{3} / 12\left(1-v^{2}\right)$ is the bending modulus. From (3) (5) and $w_{0}$ definition, one can obtain the plastic dissipation $\Gamma^{P}$ through calculating the area of the moment-curvature curves loop OABCDEO shown in figure 1(a),

$$
\begin{aligned}
\Gamma^{P}=\frac{1}{2} M_{e} \kappa_{e}-\frac{1}{2} M_{B}\left(\kappa_{B}-\kappa_{0}\right) & +\left(\frac{2}{3}-\frac{2}{N+2} \gamma\right) M_{0}\left(\kappa_{e}-\kappa_{e}^{2} / \kappa_{B}\right) \\
& +\frac{2 \gamma}{(N+1)(N+2)} M_{0}\left(\kappa_{B}^{N+1} / \kappa_{e}^{N}-\kappa_{e}\right)+\frac{1}{2} B \kappa_{0}^{2} w_{0}
\end{aligned}
$$

Two cases are considered here. Firstly, the crack tip rotation angle $\theta_{\text {tip }}$ is chosen as the peeling criterion parameter. Through applying (4) and (5) to the crack tip and using (1) and (4) (8), the normalized fracture work (or peel force) can be expressed by material parameters as

$$
P(1-\cos \Phi) / \Gamma_{0}=f\left(N, v, w_{0}, \Phi, t / R_{0}, \theta_{t i p}\right)
$$

where $R_{0}=E \Gamma_{0} / 3 \pi\left(1-v^{2}\right) \sigma_{Y}^{2}$, characterizes the plastic zone size in the small scale yielding. $\Gamma_{0}$ is the interface adhesion energy. Note that in (9), the normalized fracture work is independent of the material yielding stress, $\sigma_{Y} / E$. Secondly, the crack tip opening displacement $\delta_{c}$ is taken as the peeling criterion parameters. In this case, the embedded process zone (EPZ model) is used to characterize the fracture process (Fig. 1(b)). The normalized fracture work can be dictated as,

$$
P(1-\cos \Phi) / \Gamma_{0}=f\left(N, \mathrm{v}, w_{0}, \Phi, t / R_{0}, E / \sigma_{Y}, \hat{\sigma} / \sigma_{Y}\right)
$$

where $\hat{\sigma}$ is the interfacial separation strength, $\left(\Gamma_{0}, \hat{\sigma}\right)$ are the EPZ model dominating parameters. Note that the choice of the shape parameters $\left(\lambda_{1}, \lambda_{2}\right)$ in the relation of traction-separation law has only little influence on the results [4], so in the present analysis, take $\left(\lambda_{1}, \lambda_{2}\right)=(0,1)$.

\section{General plane analysis model for peel test problems}

Considering that the BB model is hardly to capture the plastic dissipation contributed by the interface constraint effect (crack tip singularity effect), Wei and Hutchinson [4] developed another method to treat the peel test problem. Total problem is divided into two sub-problems. One is still the beam bend problem to the right of detached film. The other one is the thin film delamination problem for the attached film part, and is analyzed by adopting the general plane analysis (GPA model). The GPA model is sketched in figure 1(a). Two sub-problems are separated from section $1\left(\mathrm{x}_{1}=\mathrm{L}_{1}\right)$. The solution of the first sub-problem can be easily obtained as

$$
M_{1}=B \kappa_{0}\left\lfloor\sqrt{\left[1-\cos \left(\Phi-\theta_{1}\right)\right]\left[2 P /\left(B \kappa_{0}^{2}\right)\right]+1-w_{0}}-1\right\rfloor
$$

at section 1. Additionally, the force $(P \cos \Phi, P \sin \Phi)$ is also exerted on the section 1 . The residual curvature $\kappa_{0}$ can be calculated from the second problem solution by formula 


$$
\kappa_{0}=-\left(12 / t^{3}\right) \int_{0}^{t}\left(x_{2}-\frac{1}{2} t\right) \varepsilon_{11}^{p}\left(x_{2}\right) d x_{2}
$$

The second problem solution can be obtained by solving the following constitutive equation of steady-state peeling case

$$
\frac{\partial \sigma_{i j}}{\partial x_{1}}=\frac{E}{1+v}\left\{\delta_{i k} \delta_{j l}+\frac{v}{1-2 v} \delta_{i j} \delta_{k l}-\frac{(3 / 2) \Omega}{[1+(2 / 3)(1+v) H / E] \sigma_{e}^{2}} \sigma_{i j}^{\prime} \sigma_{k l}^{\prime}\right\} \frac{\partial \varepsilon_{k l}}{\partial x_{1}}
$$

and by carrying out a special finite element calculation $[9,10]$, where $\sigma_{i j}^{\prime}$ is deviator stress, $\sigma_{e}=\sqrt{3 \sigma_{i j}^{\prime} \sigma_{i j}^{\prime} / 2}$ is effective stress; for plastic loading $\Omega=1$, otherwise $\Omega=0$, plastic modulus $H=E\left\{(1 / N)\left(\sigma_{e} / \sigma_{Y}\right)^{1 / N-1}-1\right\}^{-1}$ obtaining from (2). The moment and force acting on the section 1 will be equivalently replaced by a linear distributed traction. The solutions can be dictated as

$$
P(1-\cos \Phi) / \Gamma_{0}=f\left(N, \mathrm{v}, w_{0}, \Phi, t / R_{0}, E / \sigma_{Y}, \hat{\sigma} / \sigma_{Y}\right)
$$

\section{RESULTS AND ANALYSES}

Figure 2 shows some parameter relations of the peel test problem using different models. The BB model result when thin film rotation at crack tip is taken as the criterion parameter is given in figure 2(a). The normalized total fracture work (or peel force) increases monotonically with decreasing the film thickness. On the other hand, with increasing the film thickness, the normalized work decreases and asymptotes to unity. Correspondingly, the plastic dissipation decreases and tends to zero. Figure 2(b) also shows the BB model results when crack tip opening displacement is taken as the criterion parameter, in this case the EPZ model has been adopted. From figure 2(b), with decreasing the film thickness, the fracture work increases first, then decreases when $t / R_{0}<2$. Specifically as film thickness tends to zero, the normalized fracture work tends to unity, i.e., the plastic dissipation tends to zero. When film thickness is large, the fracture work decreases with increasing the film thickness. For comparison, figure 2(c) shows the GPA model results. The fracture work increases with increasing the film thickness for $t / R_{0}<5$. When $t / R_{0}>6$, the fracture work asymptotes to a stable value, which is independent of the film thickness. This case corresponds to the small scale yielding state, about which the plastic zone height is much smaller than the film thickness. Obviously, from the GPA model, as the film thickness increases, the small scale yielding condition is approached. However, using the BB model this phenomenon can not appeared.

Comparison of the both model results is shown in figure 2(d) for the relation of the normalized residual curvature versus the film thickness. From figure 2(d), the residual curvature increases much more quickly with decreasing the film thickness for the BB model than for the GPA model. This can be easily interpreted from the characters of both models. For the BB model, one-dimensional stress field (bend stress field) is characterized, and the film undergoes the strong bending deformation when the film thickness is small, accompanying the strong plastic bending dissipation. When the film thickness is large, the elastic bending is dominated, and plastic dissipation tends to zero. However, for the GPA model, due to the strong constraint effect at interface, the bending deformation of the film is considerably constrained from interface. With 
increasing the film thickness, around the crack tip, an active plastic zone always exists and a small scale yielding condition is obtained.

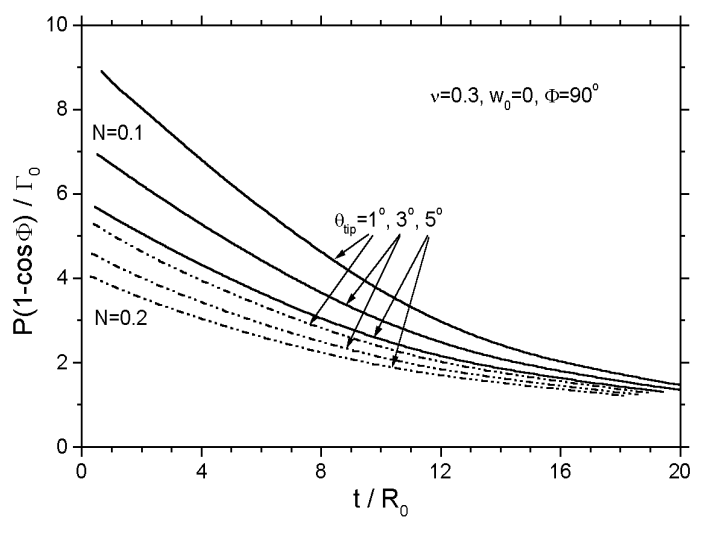

(a)

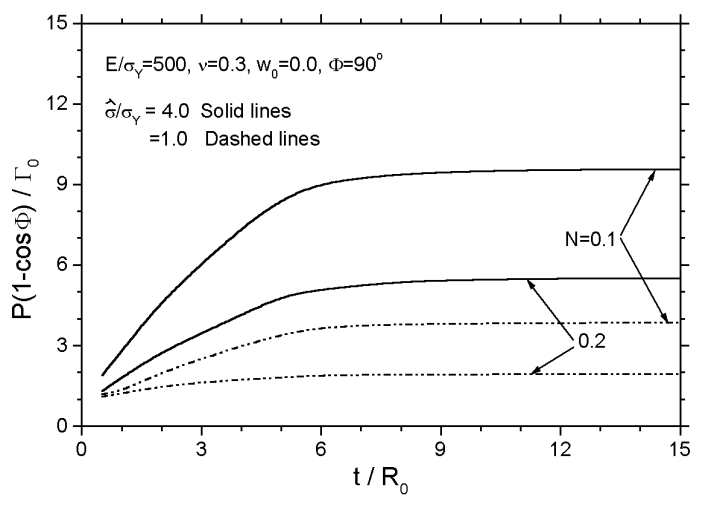

(c)

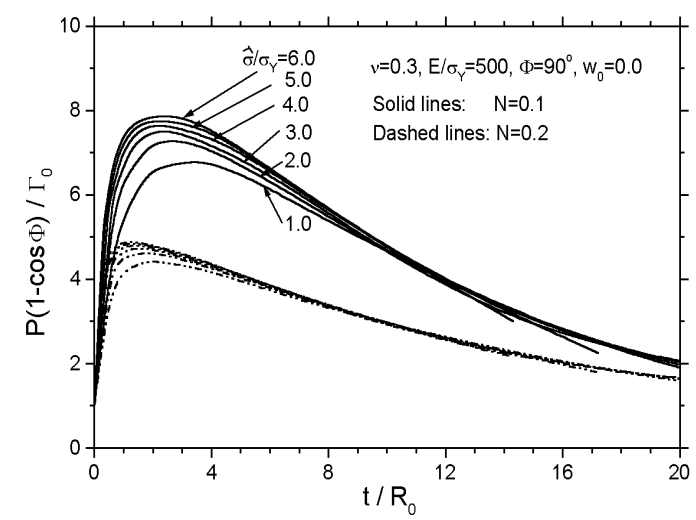

(b)

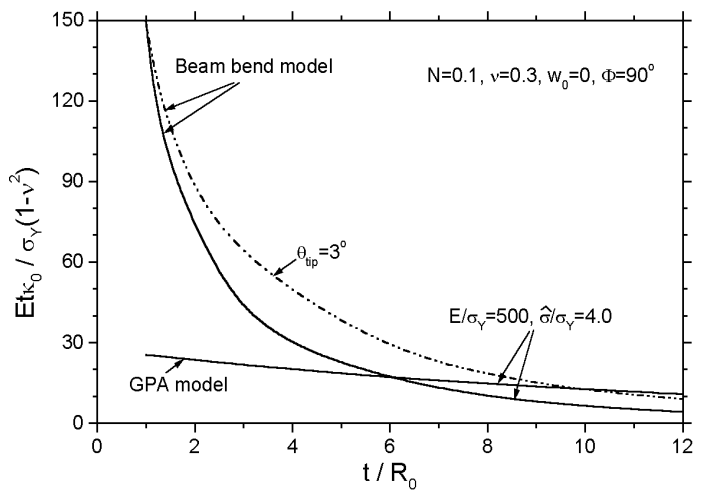

(d)

Figure 2. Fracture work and residual curvature results. For the BB model, crack tip film rotation angle as criterion parameter (a) and crack tip opening displacement as the criterion parameter (b), GPA model result (c), and (d) residual curvature results, different model result comparisons.

\section{SPLIT TEST AND ANALYSIS}

From above analyses about the peel test methods, when film is the ductile material, to measure the interfacial toughness using the peel test method becomes very difficult due to the plastic dissipation and reverse bending. From moment-curvature sketch figure shown in figure 1(a), the residual curvature $\kappa_{0}$ will be repressed due to the reverse bending after peeling. However, for split test method, sketched in figure 3(a), the reverse plastic bending doesn't take place, and the residual curvature $\kappa_{0}$ in this case is a measurable parameter. So one may obtain the interface toughness for the ductile film case through the split test method.

In the present research, the GPA model is adopted to analyze the split test, the normalized residual curvature changing with the split head angle is plotted in figure 3(b). Simultaneously, 
the result of a wedge-loaded experiment for $\mathrm{Al}$ alloy double-cantilever beam is also shown in the figure for comparison. From figure 3(b), analysis result is consistent with the experiment results [8] when the wedge tip curvature radius has a corresponding relation with the split angle.

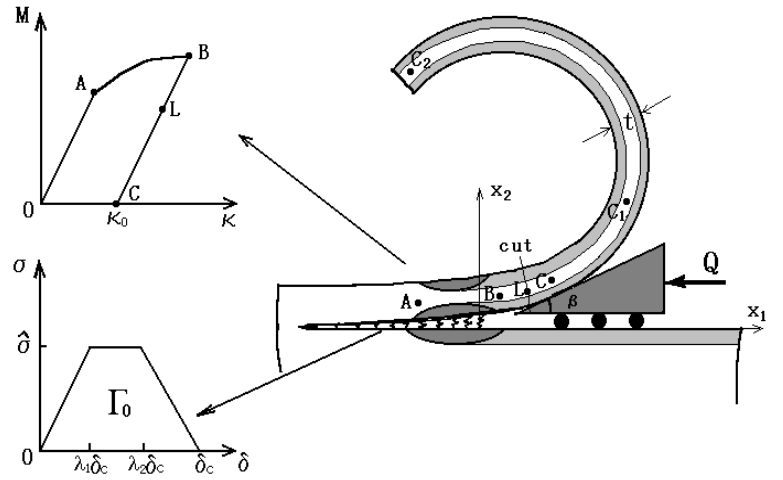

(a)

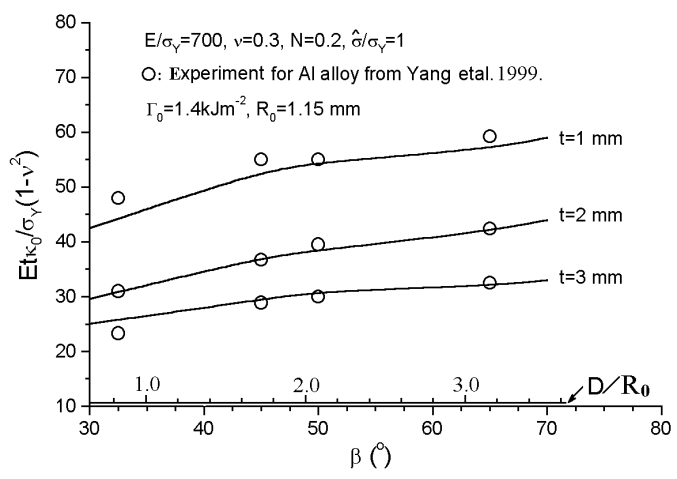

(b)

Figure 3. Split test sketch(a) and the predicted residual curvature comparing with experiment(b).

\section{CONCLUSIONS}

Conventionally used models in the peel test analysis for the ductile film/ceramic substrate system have been assessed in the present research. From the research, one can find that although the BB model captures the plastic dissipation phenomenon, however it is much sensitive to the selections of the peeling criterion parameters and it overestimates the plastic bend dissipation. Comparing with the BB model, the GPA model is strict, however it is still difficult to use this model combining with the peeling experiments for measuring the interfacial toughness. Split test may be a candidate for solving the problem due to an additional measurable parameter provided.

\section{ACKNOWLEDGMENTS}

This work was supported by the National Science Foundation of China by Grants 19891180 and 19925211, and jointly supported by the Chinese Academy of Sciences through "Bai Ren Plan".

\section{REFERENCES}

1. H. Asai, N. Iwase, and T. Suga, IEEE Trans. on Adv. Packaging. 24, 104 (2001).

2. R. Feliu-Baez, H. E. Lockhart and G. Burgess, Packaging Tech. Sci., 14, 63 (2001).

3. K.S. Kim and N. Aravas, Int. J. Solids Struct., 24, 417 (1988).

4. Y. Wei and J. W. Hutchinson, Int. J. Fracture, 93, 315 (1998).

5. A. J. Kinloch, C. C. Lau and J. G. Williams, Int. J. Fracture, 66, 45 (1994).

6. A. K. Moidu, A. N. Sinclair and J. K. Spelt, J. Testing Evaluation, 23, 241 (1995).

7. Y. B. Park, I. S. Park and J. Yu, Mater. Sci. Eng., A266, 261 (1999).

8. Q. D. Yang, M. D. Thouless and S. M. Word, J. Mech. Phys. Solids, 47, 1337 (1999).

9. R. H. Dean and J. W. Hutchinson, Quasi-static steady crack growth in small scale yielding. In Fracture Mechanics, ASTM STP 700, American Society for Testing Materials, 383 (1980).

10. Y. Wei and J. W. Hutchinson, Int. J. Fracture, 95, 1 (1999). 